\title{
Endoscopic fenestration at the splenial-habenular junctional area for symptomatic cavum and tumor at the foramen of Monro: case reports and anatomical review
}

\author{
Zamzuri Idris, MD, Jason Raj Johnson, MD, and Jafri Malin Abdullah, MD, PhD \\ Center for Neuroscience Service and Research and Department of Neurosciences, School of Medical Sciences, Universiti Sains \\ Malaysia, Kelantan, Malaysia
}

\begin{abstract}
The splenial-habenular junctional area is an alternative site for neuroendoscopic fenestration to divert CSF flow into the quadrigeminal cistern in cases in which endoscopic third ventriculostomy is not amenable. In some patients with obstructive hydrocephalus, the splenium of the corpus callosum can be elevated from the habenular complex. This exposes the membranous connection between the splenium and habenula, which can be fenestrated to divert the CSF flow into the quadrigeminal cistern. This technique can be performed in patients in whom the foramen of Monro or the third ventricle is blocked by a lesion. Here, the authors present 3 complex cases that were managed by neuronavigation-guided transventricular transcavum endoscopic fenestration of the splenial-habenular junctional area. These cases may increase the knowledge and understanding of the anatomy of this region.
\end{abstract}

http://thejns.org/doi/abs/10.3171/2014.9.JNS132683

KEY WORDS splenial-habenular junctional area; endoscopic fenestration; neuronavigation; surgical technique; anatomy

$\Lambda$ NEUROENDOSCOPIC approach to managing intraventricular lesions has been accepted as a minimally invasive surgical technique. ${ }^{6-8,10,11}$ Minimal surgical opening is required, thereby causing the least amount of injury to the brain structures. When combined with intraoperative neuronavigational image guidance, this approach allows the neurosurgeon to deal with more complex cases. ${ }^{1,4,5}$ Such complex cases are lesions or tumors within the third ventricle, which totally occlude the foramen of Monro. In emergency situations, such as obstructive hydrocephalus, the patient needs to be treated urgently. Endoscopic fenestration is our preferred method to divert the CSF because it allows us to obtain tissues for histopathological diagnosis in the same setting. In these circumstances, the splenialhabenular junctional area is a possible region to fenestrate. This identified anatomical region seems to open up and expose the thin arachnoid membranes in some cases due to obstructive hydrocephalus. With neuronavigation-guided endoscopic technique, this area can be approached from within the lateral ventricle to the quadrigeminal or tecti cistern. To our knowledge, this approach has not been described in current literature.

\section{Case Reports}

All 3 patients reported on in this study were managed in accordance and compliance with the local institutional practice regulations. The patient and family members were counseled regarding the surgical procedure, and the potential neurological and vascular complications were explained. In all situations, the patient or the family members fully understood the risks and benefits of this procedure before giving consent.

\section{Case 1}

History and Examination

An 8-year-old boy presented with a clinical history of throbbing and persistent headache, somnolence, and frequent drop attacks. Neurological examination findings were normal. Brain MRI (Fig. 1A-C) revealed a midline 
intraventricular cavum septum pellucidum and a cavum Vergae cyst. The significant feature of this lesion was the bulging of its wall and traction effect onto the corpus callosum. The important variant of the patient's brain anatomy was the presence of membranous layers connecting the lower splenium to the habenular area. Based on the patient's history and MRI findings, he was scheduled for endoscopic ventriculocystocisternostomy.

\section{Operation}

Infrared-based neuronavigation (Medtronic StealthStation TREON cranial software, Medtronic, Inc.) rigid endoscopic surgery was performed with the patient in the supine position and his head fixed. The optimum location for the bur hole and trajectory was planned based on the neuronavigational brain MR images (Fig. 2A). Endoscopic fenestration of the thickened cavum cyst wall (Fig. 2B) and the membranous layers (Fig. 2C and D) connecting the lower splenium to the habenular complex was performed using a blunt dissection with the aid of an off-mode bipolar probe. An on-mode bipolar probe was used sparingly to coagulate the edges of the fenestrated wall or membranes. A 4-F Fogarty catheter was used to enlarge the stoma (Fig. $2 \mathrm{E})$. Figure $2 \mathrm{~F}$ shows the end result of the procedure.

\section{Postoperative Course}

The presurgical symptoms improved and gradually disappeared a few days after surgery. Additional MRI of the brain showed marked differences on the pre- (Fig. 1A-C) and postsurgery (Fig. 1D-F) images. During clinic followup at 3 months, the patient was well and active and did not have any symptoms of increased intracranial pressure (ICP). Additional MRI performed at 3 months showed no hydrocephalus.

\section{Case 2}

\section{History and Examination}

A 7-year-old girl presented with symptoms of raised ICP with associated features of Parinaud and diencephalic syndromes. The physical examination revealed a cachectic-looking girl and presence of marked bilateral papilledema. Brain MRI disclosed a large tumor completely occupying the third ventricle with extension into the sellar region. Both foramina of Monro were blocked, and there was marked obstructive hydrocephalus (Fig. 3A and $\mathrm{B}$ ). The tumor markers $\alpha$-fetoprotein and $\beta$-human chorionic gonadotropin were both markedly elevated (more than $1000 \mu \mathrm{g} / \mathrm{L}$ ). The patient's pituitary profile revealed panhypopituitarism and she was treated accordingly. One of the salient features of the patient's brain MRI study was again the presence of a membranous layer connecting the lower splenium with the habenular complex (Fig. 3A, B, and G). She was scheduled to undergo neuronavigation-guided endoscopic surgery to divert the CSF into the quadrigeminal cistern without any attempt made to biopsy this highly vascular tumor, which had elevated tumoral markers.
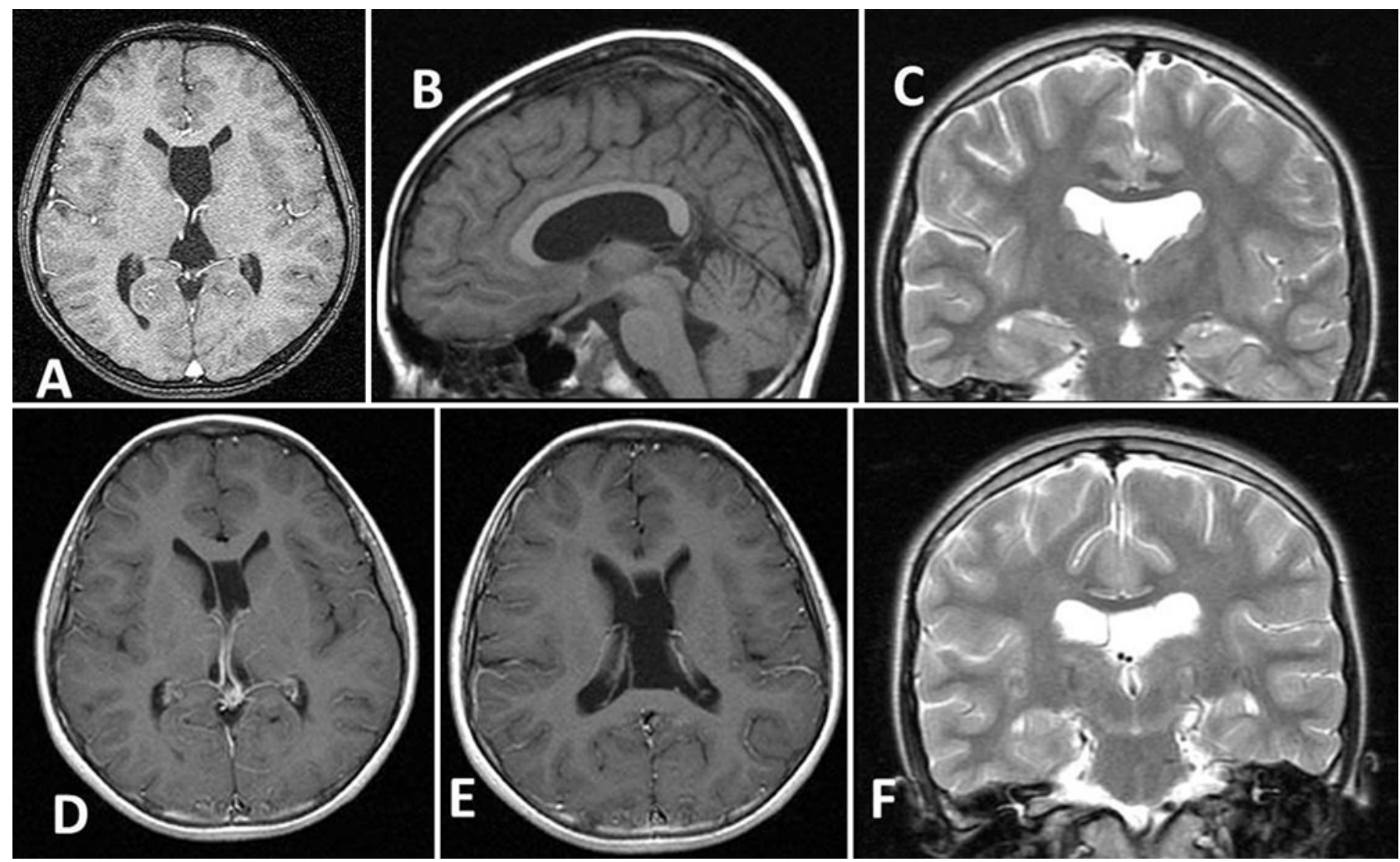

FIG. 1. Case 1. Preoperative axial (A), sagittal (B), and coronal (C) MR images showing the presence of a midline intraventricular cavum septum pellucidum and a cavum Vergae cyst. Postoperative axial (D and E) and coronal (F) MR images showing the shrunken cyst. 

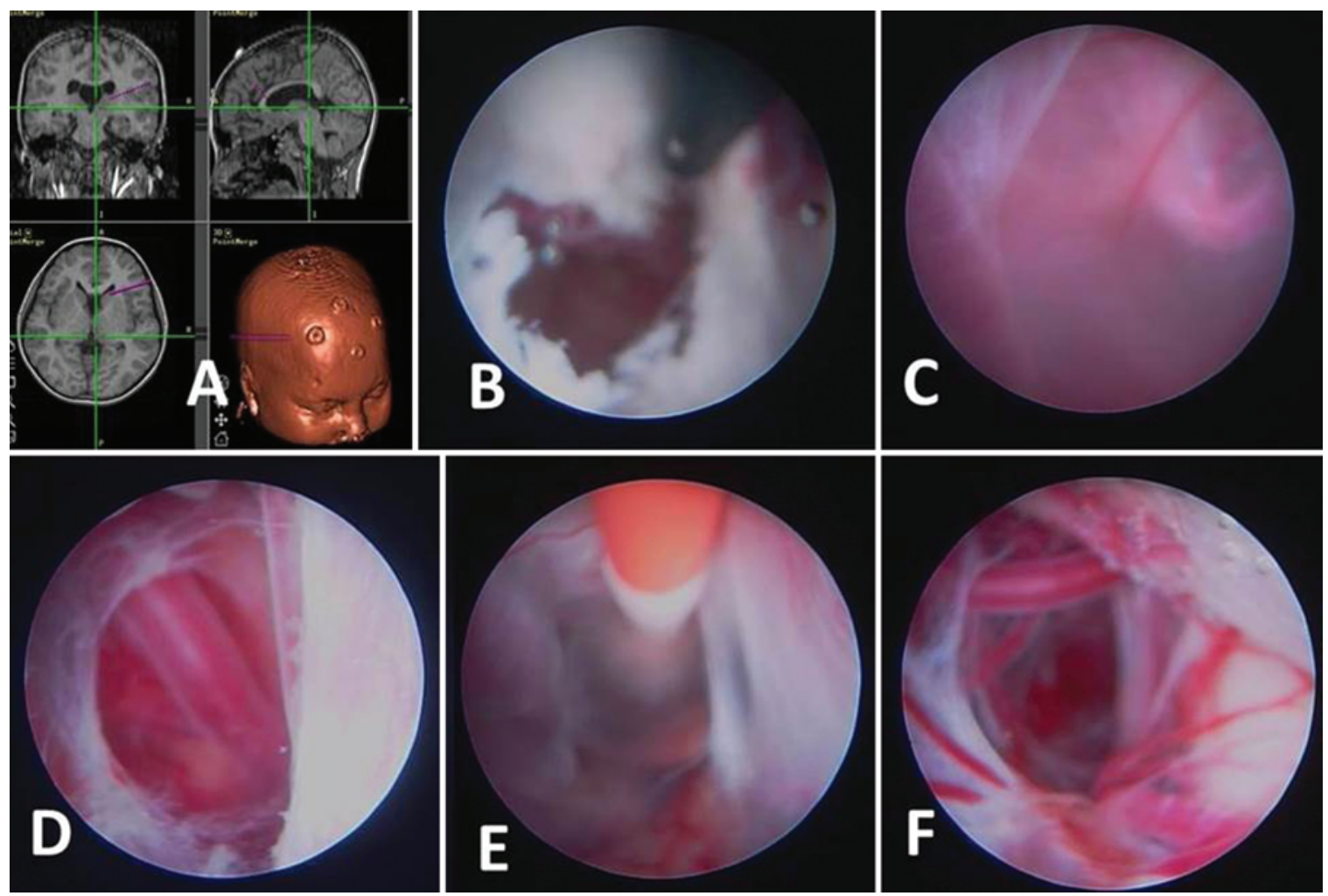

FIG. 2. Case 1. A: The location for bur hole and trajectory is planned based on neuronavigation findings. B: The cavum wall is fenestrated endoscopically. C: The membranous layer at the splenial-habenular junction. D: The membranous layer is then fenestrated by using the bipolar tips. E: A 4-F Fogarty catheter is used to dilate the stoma. F: After fenestration and dilation of the membranous layer, the vessels in the splenial-habenular area are clearly visible. Figure is available in color online only.

\section{Operation}

Infrared-based neuronavigation-guided rigid endoscopic surgery was again performed in the supine position with the head fixed. The highest and best bur hole position and trajectory were selected based on the neuronavigational brain images (Fig. 3B). Once the endoscope was advanced into the right lateral ventricle, a septostomy was performed (Fig. 3C) and further identification of the intended fenestration area was done with neuronavigation and by slightly lowering the illumination light to enhance the membranous feature at the splenial-habenular junction. This layer was incised with off-mode bipolar probe tips, and a blunt dissection was carried out until the arachnoid layers were identified. These arachnoid layers were further fenestrated until the upper cerebellum, vessels, and tentorial edge came into view (Fig. 3D).

\section{Postoperative Course}

The symptoms of raised ICP disappeared and radiological features of hydrocephalus gradually resolved (Fig. $3 \mathrm{E}$ [preoperative axial MRI brain] and $3 \mathrm{~F}$ [postoperative CT brain]). Figure $3 \mathrm{G}$ shows the stoma site. Early consultation with an oncologist was made after surgical recovery for combined radio- and chemotherapies. Additional scanning was performed 2 weeks postoperatively and showed resolution of hydrocephalus. The patient underwent follow-up in the neurosurgical clinic every 3 months, and no signs and symptoms of increased ICP were noted.

\section{Case 3}

History and Examination

A 27-year-old woman presented with complaints of progressive blurring of vision and raised ICP symptoms for 2 months. Neurological examination revealed reduced visual acuity, left inferotemporal quadrantanopia, and bilateral papilledema. Brain MRI showed the presence of a lobulated heterogeneous lesion arising from the foramen of Monro that extended to the right lateral ventricle. The septum pellucidum was curving to the left side (Fig. 4A).

\section{Operation}

The patient refused craniotomy and preferred a minimally invasive procedure. An endoscopic biopsy and CSF diversion to the quadrigeminal cistern via the splenial-habenular junction was made. Figure 4B shows the planned trajectory and the position for the right entry bur hole. Postoperative MRI showed no hydrocephalus, and the previously curving septum pellucidum returned back to its normal anatomical position (Fig. 4C). On histopathological examination the lesion was found to be a central neurocytoma (WHO Grade II).

\section{Postoperative Course}

After the endoscopy procedure, the patient's headache and vomiting resolved. The patient was referred to the oncology service for further management. At follow-up, the 

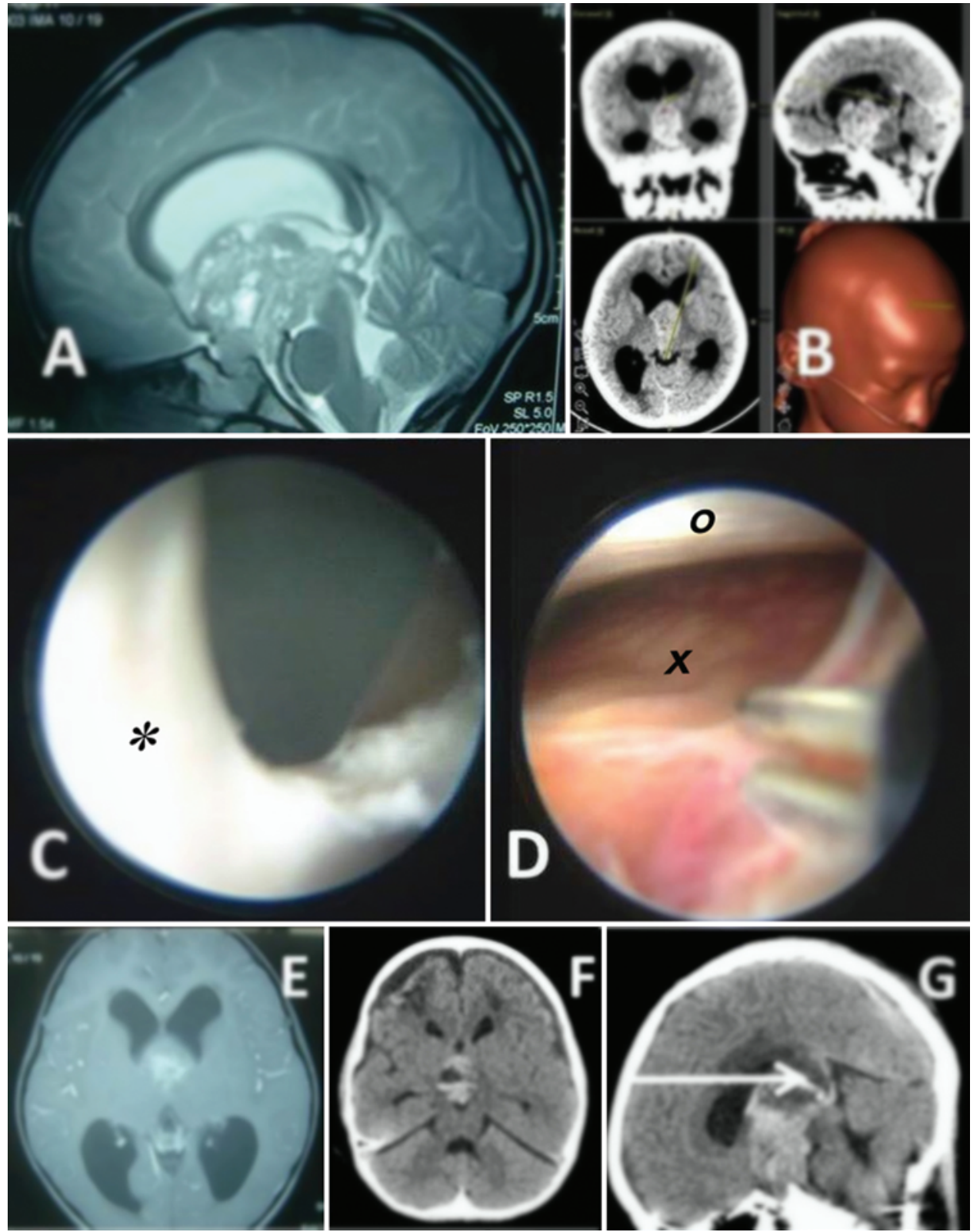

FIG. 3. Case 2. A: Sagittal MR image showing the presence of a tumor that occupies the third ventricle and causes hydrocephalus. There is a membranous layer connecting the lower part of splenium with the habenular complex. B: Bur hole and trajectory planning. C: The septostomy is performed and the opening of splenial-habenular junctional arachnoid membrane can be seen (asterisk). D: The membranous layer is fenestrated with off-mode bipolar probe blunt dissection until the upper cerebellum ( $x$ ) and tentorial edge (o) is visualized. E: Preoperative axial brain MR image showing the presence of obstructive hydrocephalus. F: Postoperative CT scan showing resolved hydrocephalus. This image was obtained prior to the commencement of radiotherapy. G: Sagittal brain CT scan showing the site of the endoscopic fenestration at the splenial-habenular junction (arrow). Figure is available in color online only.

patient was noted to be well. Additional MRI performed at 3 months and 1 year did not show presence of hydrocephalus. At subsequent clinic review, the patient did not have any symptoms of raised ICP.

\section{Discussion}

\section{Splenial-Habenular Junctional Area}

The splenial-habenular region is bounded superiorly by the splenium of the corpus callosum and inferiorly by the habenular complex, which connects both sides of the habenular nuclei. These nuclei are components of epithalamus, which in turn is a subdivision of the diencephalon. The habenular nuclei receive fibers from the striae medullaris and project via the habenulo-interpeduncular tract (fasciculus retroflexus of Meynert) to the interpeduncular nucleus of the midbrain. Basically, it forms part of a neural network that includes the limbic and olfactory systems, which are concerned with mechanisms of emotion and behavior. The pineal gland is located just caudal to 

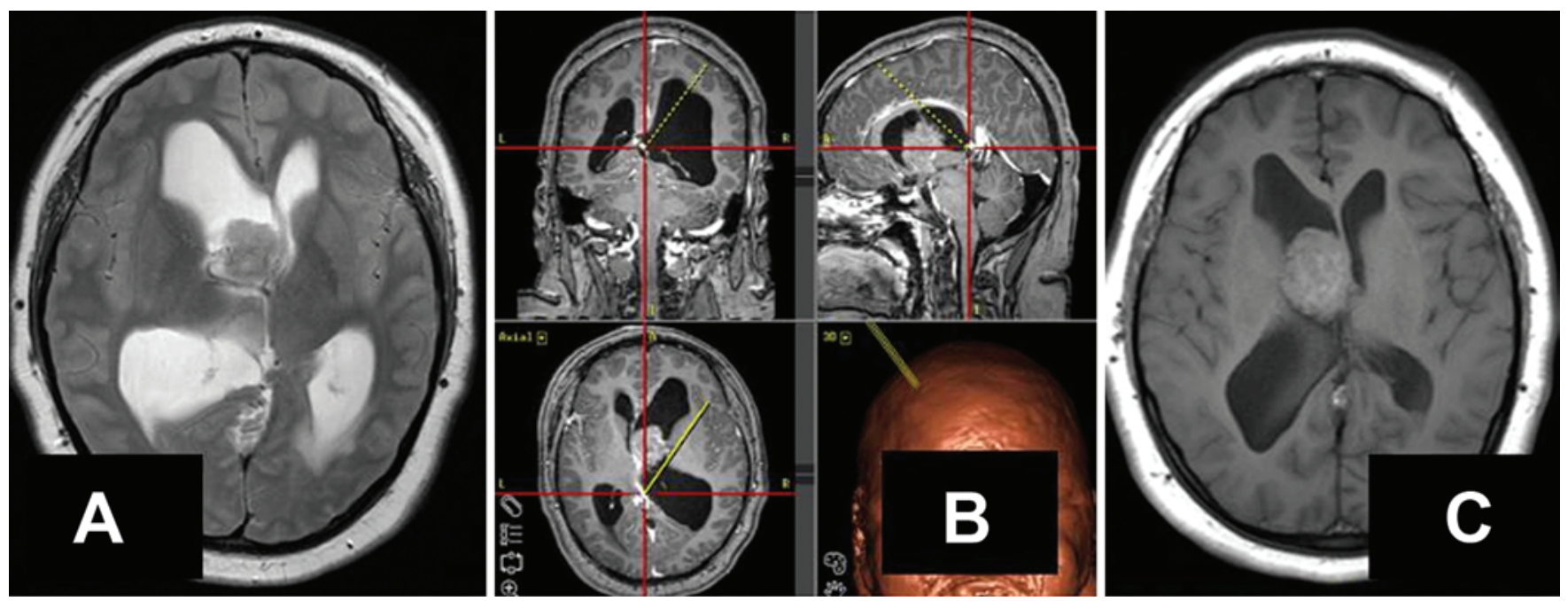

FIG. 4. A: Axial MR image showing a lobulated tumor arising from the foramen of Monro and extending into the right lateral ventricle, causing obstructive hydrocephalus. B: Neuronavigational planning. The dotted yellow line shows the planned trajectory of the endoscope. The crossed red lines represent the exact location on the splenial-habenular junctional membrane that will be fenestrated C: Postoperative MR image showing the straight septum pellucidum. Figure is available in color online only.

the habenular commissure, and the posterior commissure is situated below the pineal gland. There are arachnoid membranous connections from the splenium of the corpus callosum to the habenular complex. In some patients with obstructive hydrocephalus, secondary to a large lesion or tumor at the foramen of Monro or third ventricle, there could be an elevation of the splenium from the habenular complex, which causes stretching of these membranes. This creates an alternative site for fenestration to divert the CSF into the quadrigeminal cistern, mainly because the conventional endoscopic third ventriculostomy cannot be performed in view of total blockage at the foramen of Monro or third ventricle. The anatomical features of the splenial-habenular junctional area and cavum are depicted in Fig. 5.

\section{Cavum Anatomy}

The cavum septum pellucidum, cavum Vergae, and cavum velum interpositum are 3 cava that can be seen in humans. These are cavelike structures that are formed by the separation of their 2 leaflets. The cavum septum pellucidum and cavum Vergae are situated above the fornix, while the cavum velum interpositum is below the fornix. The cavum septum pellucidum is located anterior to the foramen of Monro, while the cavum Vergae is located posterior to the foramen of Monro. The cavum septum pellucidum (cavum septum) is a vertical membrane that connects the corpus callosum to the columns of the fornix and separates the 2 lateral ventricles. This septum has right and left leaves, where each is a part of the medial border of the lateral ventricle. In common variants, the cavum septum pellucidum is a wide and patent cavity, which often remains asymptomatic. In early literature, ${ }^{2}$ this cavity was considered as the fifth cerebral ventricle. The posterior extension of the cavum septum and lying posterior to the columns of fornix is known as the cavum Vergae and was considered as the sixth cerebral ventricle. In actual fact, these structures are not ventricles because they are not lined by ependymal cells and do not contain choroid plexus. ${ }^{9}$

The anatomical boundaries of the cavum septum are the lateral ventricular system (medial hemispheric wall) laterally, the anterior limb and pillars of the fornix posteriorly, and the anterior commissure and the rostrum of the corpus callosum inferiorly; the roof is formed by the corpus callosum. ${ }^{3}$ The cavum Vergae is bordered anteriorly by the posterior border of the nonobliterated or obliterated cavity of the cavum septum (septum pellucidum). The nonobliterated cavum septum cavity is normally in communication with the cavum Vergae through Verga's aqueduct. The inferior boundary of the cavum Vergae is the body and diverted part of the fornices and the superior and posterior boundaries are the corpus callosum..$^{12}$ Therefore, the posterior-end cavity takes the shape of a pyramid with its base lying posteroinferiorly (due to the diverted and downward Y-shaped fornices). This base lies in the area between the splenium and the habenular complex.

The cavum velum interpositum is a triangular cavity located in between the roof of the third ventricle and the body of the fornices. This cavity develops as a result of abnormal separation of the limbs of the fornix. It tends to be large in infants and becomes small beyond the age of 2 years. In contrast to the cavum septum pellucidum and the cavum Vergae, which can communicate with the ventricular system and arachnoid space, the cavity of the velum interpositum can communicate with only the subarachnoid space. Whether the cavum cavities are intra- or extracerebral structures is a matter of debate. From our endoscopic observation, the cavum cavities are extracerebral structures that are further separated from the quadrigeminal or tecti cisterns by the posteriorly located arachnoid layers.

These 3 illustrated cases highlight the importance of understanding the anatomy at this region. The first case highlights the typical features of the symptomatic cavum septum and the cavum Vergae cyst, which requires surgi- 

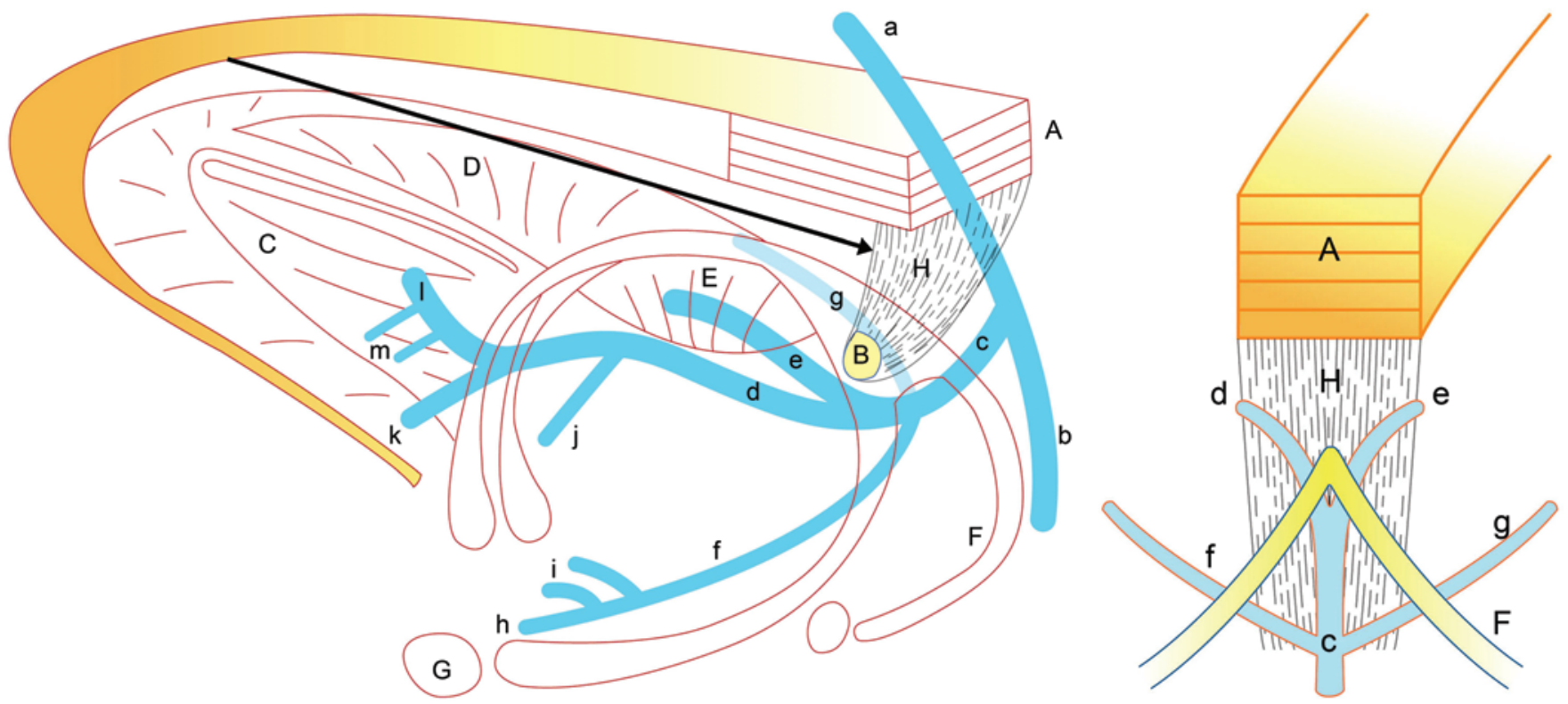

FIG. 5. Left: Illustration depicting the location of the cavum septum pellucidum, cavum Vergae, and cavum velum interpositum. The membrane connecting the splenium of the corpus callosum and the habenular complex is also shown, with the deep venous anatomy and relationship to these structures. The labeled structures are as follows: inferior sagittal sinus (a); straight sinus (b); vein of Galen (c); left internal cerebral vein (d); right internal cerebral vein (e); left basal vein of Rosenthal (f); right basal vein of Rosenthal (g); deep middle cerebral vein (h); insular veins (i); septal vein (j); caudate vein (k); thalamostriate vein (l); transverse caudate veins $(\mathrm{m})$; splenium of the corpus callosum $(\mathrm{A})$; habenular complex (B); cavum septum pellucidum (C); cavum Vergae (D); cavum velum interpositum (E); crus of fornix $(F)$; amygdala $(G)$; and splenial-habenular arachnoid membrane $(H)$. The arrow represents the endoscopic trajectory, which transgresses the ventricle, cavum, and finally the membrane (septostomy is normally required in most cases). Right: Illustrated dorsal view of the splenial habenular arachnoid membrane and its adjacent deep venous and neural structures. Labeled structures are described in the left panel. Copyright Mohamed Zafrualam Muhd. Zain. Published with permission. Figure is available in color online only.

cal intervention. Brain MRI revealed bulging of their walls and elevation of the splenium, which caused widening of the posterior wall (base of the pyramid) of the cavum Vergae, and it was separated from the quadrigeminal or tecti cisterns by only thin layers of arachnoid membrane. For the management of a cystic cavum, communicating the cyst cavity with the ventricular cavity (ventriculocystostomy) would allow for the cyst fluid to drain. However, this may not be adequate. Hence, by performing a fenestration of the splenial-habenular junctional membrane, an additional route of CSF outflow is established via the quadrigeminal cistern (ventriculocystocisternostomy). The second and third cases highlight the importance of appreciating and understanding the anatomy of the cavum cavity and also remind us to thoroughly inspect the sagittal brain images and pay close attention to the splenial-habenular junctional area. In our second case, the patient had a massive tumor filling the third ventricular space completely that caused obstruction at the foramen of Monro bilaterally. In this case, endoscopic third ventriculostomy was not possible as the floor of the third ventricle is not accessible. Therefore, the splenial-habenular junctional fenestration provides an alternative route for CSF diversion. In some cases of large third ventricular or pineal region tumors with associated hydrocephalus, the splenium of the corpus callosum can be elevated superiorly and the habenular region might be displaced further caudally, which both can cause stretching of the membranous area at the splenial-habenular junction. In these circumstances, the widened and stretched membrane at the splenial-habenular junctional area could be an alternative site to divert the CSF (as illustrated in Cases 2 and 3).

\section{Conclusions}

We have highlighted the importance of understanding the anatomy of the symptomatic cavum septum and the cavum Vergae, and the asymptomatic cavum Vergae cavity. Thorough understanding of the anatomy may help neurosurgeons to navigate and fenestrate the splenial-habenular junctional area safely in creating a new CSF diversion pathway and to traverse the quadrigeminal cistern from the lateral ventricular approach in some selected cases.

\section{References}

1. Alberti O, Riegel T, Hellwig D, Bertalanffy H: Frameless navigation and endoscopy. J Neurosurg 95:541-543, 2001 (Letter)

2. Auer RN, Gilbert JJ: Cavum vergae without cavum septi pellucidi. Arch Pathol Lab Med 106:462-463, 1982

3. Born CM, Meisenzahl EM, Frodl T, Pfluger T, Reiser M, Möller HJ, et al: The septum pellucidum and its variants. An MRI study. Eur Arch Psychiatry Clin Neurosci 254:295302, 2004

4. Chernov MF, Kamikawa S, Yamane F, Ishihara S, Kubo O, Hori T: Neurofiberscopic biopsy of tumors of the pineal 
region and posterior third ventricle: indications, technique, complications, and results. Neurosurgery 59:267-277, 2006

5. Cipri S, Gangemi A, Cafarelli F, Messina G, Iacopino P, Al Sayyad S, et al: Neuroendoscopic management of hydrocephalus secondary to midline and pineal lesions. J Neurosurg Sci 49:97-106, 2005

6. Idris Z, Ghani AR, Idris B, Muzaimi M, Awang S, Pal HK, et al: Neuronavigation-guided endoscopic management of a pineal region tumour with obscured floor of the third ventricle: case report. Minim Invasive Neurosurg 54:125-127, 2011

7. Idris Z, Hallaert G, Vanhauwaert D, Kalala JP, Dewaele F, Baert E, et al: Frameless neuronavigation-guided endoscopic total en-bloc removal of a third ventricular colloid cyst: a case report on surgical technique. Minim Invasive Neurosurg 51:173-177, 2008

8. Kim IY, Jung S, Moon KS, Jung TY, Kang SS: Neuronavigation-guided endoscopic surgery for pineal tumors with hydrocephalus. Minim Invasive Neurosurg 47:365-368, 2004

9. Osborn AG, Preece MT: Intracranial cysts: radiologicpathologic correlation and imaging approach. Radiology 239:650-664, 2006

10. Prat R, Galeano I: Endoscopic biopsy of foramen of Monro and third ventricle lesions guided by frameless neuronavigation: usefulness and limitations. Clin Neurol Neurosurg 111:579-582, 2009
11. Schroeder HW, Wagner W, Tschiltschke W, Gaab MR: Frameless neuronavigation in intracranial endoscopic neurosurgery. J Neurosurg 94:72-79, 2001

12. Tubbs RS, Krishnamurthy S, Verma K, Shoja MM, Loukas M, Mortazavi MM, et al: Cavum velum interpositum, cavum septum pellucidum, and cavum vergae: a review. Childs Nerv Syst 27:1927-1930, 2011

\section{Author Contributions}

Conception and design: Idris, Johnson. Acquisition of data: Idris, Johnson. Analysis and interpretation of data: Johnson. Drafting the article: Johnson. Critically revising the article: Idris, Abdullah. Approved the final version of the manuscript on behalf of all authors: Idris. Administrative/technical/material support: Abdullah. Study supervision: Abdullah.

\section{Correspondence}

Zamzuri Idris, Department of Neurosciences, School of Medical Sciences, Hospital Universiti Sains Malaysia, 16150 Kubang Kerian, Kelantan, Malaysia. email: neuroscienceszamzuri@ yahoo.com. 